\title{
Does Kinesiophobia Effect One Day Physical Activity Behaviour and Functionality of Young People with Ankylosing Spondylitis?
}

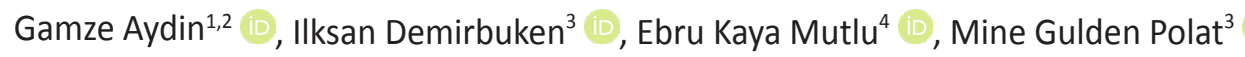 \\ ${ }^{1}$ Istanbul University-Cerrahpasa, Institute of Graduate Education, Department of Physiotherapy and Rehabilitation, Istanbul, Turkey \\ ${ }^{2}$ Istanbul Okan University, Faculty of Health Sciences, Division of Physiotherapy and Rehabilitation, Istanbul, Turkey \\ ${ }^{3}$ Marmara University, Faculty of Health Sciences, Division of Physiotherapy and Rehabilitation, Istanbul, Turkey \\ ${ }^{4}$ Istanbul University-Cerrahpasa, Faculty of Health Sciences, Division of Physiotherapy and Rehabilitation, Istanbul, Turkey \\ Correspondence Author: Gamze Aydin \\ E-mail: gmzetsn@gmail.com
}

Received: $28.02 .2019 \quad$ Accepted: 21.12 .2019

\begin{abstract}
Objective: The aim of this study was to investigate the relationship between kinesiophobia, physical activity behaviour and functionality in patients with ankylosing spondylitis (AS).

Methods: This study included 57 participants (mean age, $30.8 \pm 5.19$ years) in 2 groups of AS and healthy controls. One day physical activity behaviour was evaluated with tri-axial accelerometer. Tri-axial accelerometer was recorded physical activity domains and intensities for 24 hours. Participants completed clinical questionnaires assessing pain, kinesophobia, disease activity, and functionality. Kinesiophobia and pain were quantified by the Tampa Scale for Kinesiophobia and Short-Form McGill Pain Questionnaire. Function and disease activity were rated by using the Bath Ankylosing Spondylitis Functional Index, and Bath Ankylosing Spondylitis Disease Activity Score.

Results: There was no correlation between kinesiophobia and one day physical activity behaviour in AS patients group. There was a negative correlation between high physical activity level and disease activity $(p<0.05, \mathrm{r}:-.519)$ and there was a negative correlation between high and moderate physical activity levels, and functionality ( $p<0.05$, r:-.555 $r$ :-395, respectively).

Conclusion: According to the results of this study showed that; there was a relationship between disease activity, functionality, and one day physical activity behaviour. On the other hand, there was not any relation between pain intensity, kinesophobia and data obtained from accelerometer. Further studies with larger sample size and longer time periods should be carried out to reveal physical activity behaviours and its related factors in AS patients who will survive with this disease for years.
\end{abstract}

Keywords: ankylosing spondylitis, disease activity, functionality, kinesophobia, physical activity

\section{INTRODUCTION}

AS is a rheumatic disease characterized by inflammation, pain, and stiffness especially in the spinal column and sacroiliac joints $(1,2)$. The disease is prevalent between the ages of 20 and 30 which is an important period in lifespan for career stages and performing physically active life (3).

AS restrains individual's physical capacity and results in reduced participation in physical activity in their daily living due to restricted respiratory functions, pain, limitation of joints and spinal motions (4). Since it is well known that participation in regular physical activity can prevent chronic systemic diseases and improve musculoskeletal health, it is important to encourage AS patients to avoid getting into this vicious cycle (limited physical activity due to symptoms and exacerbated symptoms due to limited physical activity) (5). Therefore, it is a prerequisite to examine the factors that cause physical activity limitation, especially in adult AS patients who will survive with this disease for years.
Recently, some factors that influence physical activity have been identified by few researches (6-8). Disease activity, pain, depression, flexibility and respiratory functions are some of them. But there are few studies investigating the kinesiophobia which is defined by Kori et al,'a fear of movement resulting from a feeling of vulnerability to painful injury or reinjury' $(9,10)$. It is assumed to be pain-related experiences, combined with kinesiophobia, may be more disabling than pain itself.

Functionality in daily living is an other crucial factor to maintain qualified life especially in young ages. Mobility restrictions of spine and pain are one of the limiting factors for functionality. Nas et al. have revealed this relation in AS (11). But, we have not known yet if kinesiophobia affects the physical activity behaviour and functionality in AS patients.

We suggest that it is important to identify the physical activity behaviour of patients with AS at young ages, firstly. Secondly, 
factors relating with AS that cause limitations in physical activity and functionallity should be revealed to be able to overcome these obstacles. Therefore, the aim of the study was to investigate the relationship between kinesiophobia with physical activity behaviour and functionality in young patients with AS.

\section{METHODS}

\subsection{Study design and recruitment}

The study was designed as a cross-sectional observational. It was carried out between December 2015 and May 2016, with patients who were diagnosed as AS, in rheumatology clinic, in Istanbul University-Cerrahpasa, Cerrahpasa Faculty of Medicine Hospital. Forty-two AS patients were screened for possible inclusion criteria whereas thirty AS patients agreed to participate in the study and twenty-seven agematched healthy people were included into the control group. Patients with AS were recruited from a register of patients fulfilling the Newyork Classification Criteria, diagnosed by a rheumatologist at Istanbul UniversityCerrahpasa, Cerrahpasa Faculty of Medicine Hospital, Istanbul. Eight patients withdrew from the assessment and four did not attend the accelerometer assessment, leaving a total of 30 patients who completed the study (Fig 1). Controls were recruited from the healthy hospital staff member with age matched AS patients. Participants in control group did not report any pain complaint.

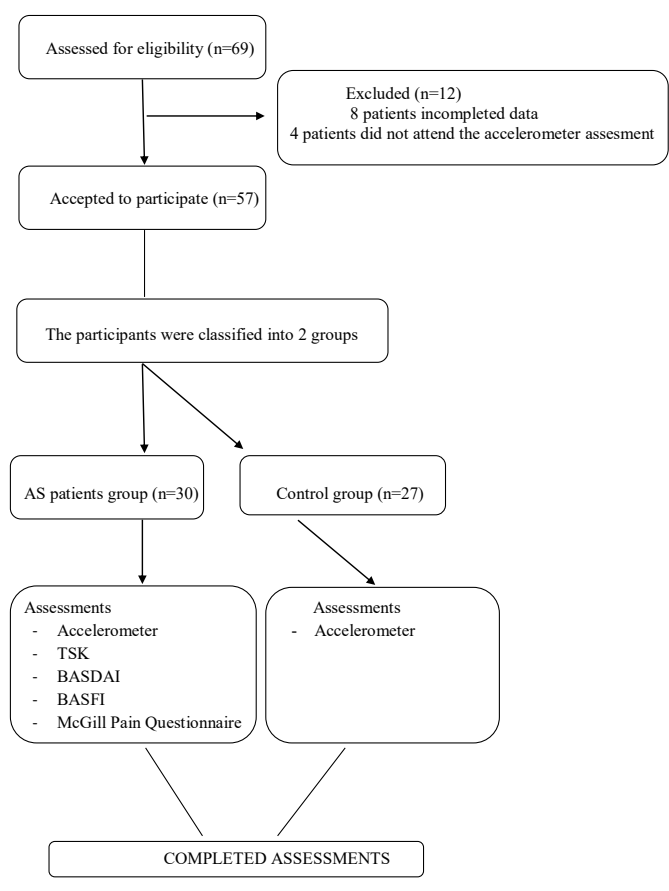

Figure 1. Design of the study

To participate in the study, subjects should have been between 20-40 years old, should have had the absence of lower extremity involvement, should have agree to participate in the study, and sign an informed consent form.
The exclusion criteria were having the cognitive disorder, the neurological deficit in lower limbs, visual, auditory, vestibular problems that could affect the independent walk. AS patients continued similar medical treatment in their routine.

Each participant who completed a recruitment screening received written and verbal explanations of the procedures to be applied. They were all asked to participate on a voluntary basis. The project observed the principles outlined in the Declaration of Helsinki of 2013, and the study protocol was approved by the Human Research Ethics Committee of Marmara University (Institutional Review Board No: 30.11.2015-4).

\subsection{Assessment}

The evaluation was comprised of questionnaires parts and accelerometer measurements. The characteristics of participants (age, gender, weight, height, employment, education, regularly exercise habit) were questioned by the Socio-Demographic Data Form. Participants were asked to complete the questionnaires, which assessed disease activity, functionality, kinesophobia, pain intensity. Physical activity level was assessed with triaxial accelerometer. Tampa Scale for Kinesiophobia (TSK) was used to measure kinesiophobia scores. The pain was evaluated with Kısa Form-McGill Pain Questionnaire (SF-MPQ), disease activity was evaluated with Bath Ankylosing Spondylitis Disease Activity Index (BASDAI), functionality was evaluated with Bath Ankylosing Spondylitis Functional Index (BASFI). All measurements were performedby the same physiotherapist for 2 groups.

\subsection{Physical activity measurement}

The one day physical activity behaviour was assessed according to the results of the triaxial accelerometer device manufactured by Maastricht University in the Netherlands (MOX). Accelerometer was recorded for 24 hours for each case, from 08:00 am until the next morning 8:00 am.

Records were uploaded to the computer program and the data was saved. A day was chosen when he/she continued any daily activities except Sunday, and he/she was moved in the middle of his/her pants belt. Participants measured minute by minute accelerations (expressed as counts) in the anteroposterior, mediolateral, and longitudinal axes of the trunk. Total sedentary time (sitting/reaching), total standing time, total light physical activity time, total moderate physical activity time, total high physical activity time were recorded (minutes per day) (12).

\subsection{Disease-related variables}

TSK consists of the 17 items that are rated using a 4-point Likert scale whereby 1 = strongly disagree and 4 = strongly agree. The total kinesophobia score is the sum of responses to all 17 items. The total score ranges from 17 to 68 points, with higher scores indicating the presence of kinesophobia. 
The TSK is a reliable and valid instrument for the assessment of kinesophobia in the Turkish population (13).

Pain severity and localization of the cases were evaluated according to the results of SF-MPQ. It consists of 15 descriptive adjectives for the pain sensation, which are selfrated by the patient (range from 0-3 points (from none to severe). The total score is the sum of the intensity values of words chosen for sensory, effective and total descriptors. The SF-MPQ includes a pain intensity measure indicated by the visual analog scale (VAS) (14). The SF-MPQ is a valid construct within the Turkish culture (15).

BASDAl was used for an assessment of the disease activity. Six questions were asked. Fatigue, pain, discomfort, swelling was questioned in the first five questions.In the sixth question, the patient described their "morning stiffness". The patients scored between 0 and 10, with 0 indicating no and 10 indicating very severe. The total BASDAI score was calculated by the average of the scores obtained from the fifth and sixth questions and the sum of all the items and dividing them by $5 .((1+2+3+4+(5+6 / 2)) / 5)$. The BASDAI is a valid construct within the Turkish culture (16).

BASFI consists of the 10 items that are rated by using a $10 \mathrm{~cm}$ visual analog scale whereby $0=$ easy and $10=$ not possible, for each activity. The score is sum of all the items and dividing them by 10 . Higher scores indicate less functionality. The Turkish version of the BASFI has been shown to be a valid and reliable scale for the assessment of functional status (17).

\subsection{Statistical analysis}

The SPSS version 21.0 for Windows was used to evaluate data and analyze descriptive statistics (frequency, mean, SD). Statistical analysis was performed at a $95 \%$ confidence level, and the statistical significance level was set as 0.05 . In the present study, conformity to normal distribution was analyzed using the Shapiro-Wilks test. Descriptive statistics were used for demographic variables. Independent sample t-test (parametric test) and Mann Whitney $U$ test (nonparametric test) were used to analyze the continuous variables. Intercorrelations between the one day physical activity behaviour, pain, kinesophobia, disease activity and functionality parameters were analyzed using by Spearman correlation analysis (specifically, $r=0.5-1.0$ was large; $r=0.30$ 0.49 was medium, and $r=0.1-0.29$ was small).

\section{RESULTS}

The comparisons of the demographic and clinical characteristics of the groups are shown in Table 1 . No significant differences were found between the groups in regards to the demographic characteristic (age, weight, height, BMI, employment, regularly exercise habits parameters) ( $p>0,05)$. The TSK score average was 46.56 $( \pm 5.41)$, the McGill pain questionnaire score average was 24.76 ( \pm 6.91$)$, the BASDAl total score average was 5.64 ( \pm 1.30), and the BASFI total score average was 3.78 of the AS patients group (Table 1). AS group's kinesiophobia levels and disease activities were found to be high. At the same time, functionality were found to be low.

Table 1. Demographic and disease-specific variables

\begin{tabular}{|c|c|c|c|}
\hline & $\begin{array}{c}\text { AS } \\
(n=30)\end{array}$ & $\begin{array}{l}\text { Controls } \\
(n=27)\end{array}$ & $p^{a}$ \\
\hline \multicolumn{4}{|l|}{ Demographic } \\
\hline Age (years) & $32.6 \pm 5.04$ & $28.9 \pm 4.73$ & 0.06 \\
\hline Female sex (n/\%) & $15(50 \%)$ & $21(77.7 \%)$ & 0.03 \\
\hline Weight (kg) & $67.3 \pm 9.53$ & $64.1 \pm 13.4$ & 0.29 \\
\hline Height $(\mathrm{cm})$ & $167 \pm 8.54$ & $166 \pm 7.30$ & 0.63 \\
\hline $\mathrm{BMI}\left(\mathrm{kg} / \mathrm{m}^{2}\right)$ & $23.9 \pm 2.69$ & $23 \pm 3.95$ & 0.28 \\
\hline Employment (n/\%) & $20(66.7 \%)$ & $17(62.9 \%)$ & 0.77 \\
\hline Education $\geq 12$ years $(n / \%)$ & $14(46.7 \%)$ & $25(92.5 \%)$ & 0.001 \\
\hline Regulary exercise habits ( $\mathrm{n} / \%$ ) & $4(13.3 \%)$ & $3(11.1 \%)$ & 0,79 \\
\hline \multicolumn{4}{|l|}{ Disease characteristics } \\
\hline $\begin{array}{l}\text { Because of AS, applying } \\
\text { to Physiotherapy and } \\
\text { Rehabilitation Units }\end{array}$ & $1(3 \%)$ & N/A & \\
\hline BASDAI & $5.64 \pm 1.3$ & N/A & \\
\hline BASFI & $3.78 \pm 1.91$ & N/A & \\
\hline McGill Pain Score & $24.76 \pm 6.91$ & N/A & \\
\hline TAMPA Kinesophobia Score & $46.56 \pm 5.41$ & N/A & \\
\hline
\end{tabular}

BMI:Body Mass Index, AS: Ankylosing Spondylitis, BASDAI Bath Ankylosing Spondylitis Disease Activity Index, BASFI Bath Ankylosing Spondylitis Functional Index, N/A: not assessed, number of participants (\%) and Mean

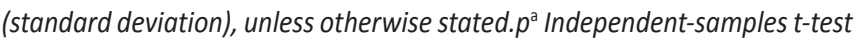

Moderate level physical activity time was lower in AS group than controls $(p<0.05)$ while there was no statistically significant difference in other domains of physical activity (total sedentary, standing, light and highlevel physical activity time) (Table 2).

Table 2. Daily physical activity behaviour in different domains, in patients with AS and controls

\begin{tabular}{|l|c|c|c|c|} 
& $\begin{array}{c}\text { AS } \\
(\mathrm{n}=30)\end{array}$ & $\begin{array}{c}\text { Controls } \\
(\mathrm{n}=30)\end{array}$ & $\mathbf{t}^{\mathrm{a}}$ & $\mathbf{p}^{\mathrm{a}}$ \\
\hline $\begin{array}{l}\text { Domains } \\
\text { (minutes) }\end{array}$ & Mean $\pm \mathrm{SD}$ & Mean $\pm \mathrm{SD}$ & & \\
\hline Sedantery & $786.7 \pm 404.5$ & $1045.7 \pm 408.2$ & -2.4 & $\mathbf{0 . 0 2 *}$ \\
\hline Standing & $377.9 \pm 175.2$ & $379.2 \pm 198.7$ & -0.02 & 0.97 \\
\hline Moderate & $64.2 \pm 45.4$ & $91.1 \pm 52.8$ & -2.07 & $\mathbf{0 . 0 4}$ \\
\hline $\begin{array}{l}\text { Domains } \\
\text { (minutes) }\end{array}$ & Mean $\pm \mathrm{SD}$ & Mean $\pm \mathrm{SD}$ & $\mathbf{z}^{\mathrm{b}}$ & $\mathbf{p}^{\mathrm{b}}$ \\
\hline Light & $9.46 \pm 5.1$ & $13.9 \pm 16.4$ & -0.32 & 0.97 \\
\hline High & $2.70 \pm 7.86$ & $5.66 \pm 13.2$ & -1.43 & 0.15 \\
\hline
\end{tabular}

SD:Standart Deviation, min:minimum, max:maximum. aIndependentsamples t-test, ${ }^{\mathrm{b}}$ Mann Whitney $\cup$ Test

Disease activity was found to be correlated with total high level physical activity time $(r:-.519, p<0.05)$. As the BASDAI total score increased, the severity of physical activity decreased. Therefore, functionality was found to be correlated with total moderate and high level physical activity time ( $p<0.05, r$ : $.555 \mathrm{r}:-.395$, respectively) (Table 3 ). When the BASFI total 
score decreased, total moderate and high physical activity time increased.

On the other hand, no correlation was found with kinesiophobia, pain and one day physical activity behaviour ( $p>0.05$ ) (Table 3).

Table 3. Comparison between results of accelerometer and TAMPA Kinesophobia Score, McGill Pain Score, BASDAI, BASFI

\begin{tabular}{|c|c|c|c|c|}
\hline $\begin{array}{l}\text { Variables } \\
\text { (AS) }(n=30)\end{array}$ & $\begin{array}{l}\text { TAMPA Kinesiophobia } \\
\text { Score }\end{array}$ & $\begin{array}{l}\text { McGill Pain } \\
\text { Score }\end{array}$ & BASDAI & BASFI \\
\hline Sedentary & $\begin{array}{l}\mathrm{rh}_{\mathrm{o}}-.109 \\
\text { p.567 }\end{array}$ & $\begin{array}{r}-.035 \\
.855\end{array}$ & $\begin{array}{l}-.206 \\
.275\end{array}$ & $\begin{array}{r}-.185 \\
.327\end{array}$ \\
\hline Standing & $\begin{array}{l}r_{o}-.018 \\
p .925\end{array}$ & $\begin{array}{r}-.205 \\
.278\end{array}$ & $\begin{array}{r}-.078 \\
.680\end{array}$ & $\begin{aligned}-.115 \\
.546\end{aligned}$ \\
\hline Light & $\begin{array}{l}\mathrm{rh}_{0} .142 \\
\mathrm{p} .454\end{array}$ & $\begin{array}{l}-.026 \\
.893\end{array}$ & $\begin{array}{l}.029 \\
.878\end{array}$ & $\begin{array}{l}-.187 \\
.321\end{array}$ \\
\hline Moderate & $\begin{array}{l}\text { rh. } .027 \\
\text { p.888 }\end{array}$ & $\begin{array}{l}-.057 \\
.765\end{array}$ & $\begin{array}{l}-, 113 \\
.551\end{array}$ & $\begin{array}{c}-.395^{*} \\
.031\end{array}$ \\
\hline High & $\begin{array}{l}\mathrm{rh}-.307 \\
\mathrm{p}, 099\end{array}$ & $\begin{array}{l}-.254 \\
.176\end{array}$ & $\begin{array}{c}-.519^{* *} \\
.003\end{array}$ & $\begin{array}{c}-.555^{* *} \\
.001\end{array}$ \\
\hline
\end{tabular}

BASDAI Bath Ankylosing Spondylitis Disease Activity Index, BASFI Bath Ankylosing, Spondylitis Functional Index, Spearman Correlations Test.

\section{DISCUSSION}

Current study examine the presence of kinesiophobia and its correlation with physical activity behaviour in patients with AS. On the basis of the our study's results, it is worthwhile to note that $96.7 \%$ AS patients in the current study had high kinesiophobia levels. Moderate level physical activity time was lower in AS group than controls. AS groups' disease activities were found to be high. There was no correlation with kinesophobia, pain and physical activity behaviour. However, there was a negative correlation between disease activity and total high level physical activity time. In addition, there was a negative correlation between functionality and total moderate and high level physical activity time, too.

Interestingly, our results showed that physical activity levels of AS patients aged 20-40 years were not affected by high kinesophobia and pain intensities. Most of the studies investigating kinesiophobia were carried out on musculoskeletal disorders and some of them involved ankylosing spondylitis, Sjögren's syndrome, fibromyalgia, osteoarthritis(specially knee) among rheumatical diseases $(10,18,19,20)$. Er G. et al, investigated relation between kinesophobia and respiratory functions in AS (20-70 years). Their study did not find a significant relationship between TSK values and respirotary functions and endurance (21). Swinnen et al, reported that TSK-11 is a promising and valid tool to assess fearful beliefs in relation to activity limitations in axial spondyloarthropathy (22). In recent study Oskay et al, examine the level of kinesiophobia and its correlation with some clinical variables in patients with AS (mean age $37.6 \pm 10$ years) (10). They found that, AS patients had high kinesiophobia level $(\geq 37)$ and there was a correlation between kinesiophobia and physical function (assesed by BASFI), but kinesiophobia was not correlated with disease activity or mobility levels. In the current study, AS group's kinesiophobia levels was found to be high but there was no correlation between kinesiophobia and one day physical activity behaviour. However, kinesophobia affects the motivation of activity negatively (23). On the other hand, in a study published in 2016, Felicio et al. found that the pain which caused disability was not associated with a kinesiophobia in lowback pain (24).

One of the most common symptoms of AS is the pain. Fongen et al. indicated that the majority of patients had more obstacles than controls while performing physical function, these were pain-related disorders such as fatigue, stiffness, and disability. They reported that the pain and disease activity could be reduced physical activity level (25). In a study, avoidance of activity was also correlated with pain and depressionon patients with chronic pain (26). However, kinesiophobia was correlated neither with pain nor with physical activity in our study, suggesting that kinesiophobia in patients with AS was not a direct obstacle because of their young ages. Even so, this potential relationship should be investigated by further studies with greater sample size.

Moderate level physical activity time was lower in AS group than controlsand AS group spent less sedentary time in a day compared to controls while there was no statistically significant difference in other domains of physical activity (total standing, light and high physical activity time) in both groups. In a recent study showed that patients with AS had similar total physical activity, compared with controls, but may avoid in higher intensities of physical activity (25). In another study, Swinnen et al. used a limb containing two axial accelerometers and thermal sensors to evaluate physical activity, between 40 adult with spondyloarthropathies (mean age $44 \pm 10$ years) and 40 healthy controls. There was no significant difference between sedentary, light and moderate physical activity levels among the groups (27). Plasqui et al. compared the physical activity levels of 25 AS patients with 25 healthy adult controls (mean age $48 \pm 11$ ) using a triaxial accelerometer, and they found no significant difference in measured parameters between groups (2). Arends et al. used a uniaxial accelerometer to evaluate daily physical activity levels in patients with AS(mean age 44 years) and reported that their study had the limitations because their tool only provided single-axial motion recordings (23). The accelerometer which we used in our study (MOX) was Tri-Axis Digital Accelerometer. Three axial movements' accelerations, anteroposterior, mediolateral and vertical, can be recorded (28). The one of the possible explanation of nonsignificant difference in one day physical activity levels between healthy and AS groups in our study could be the characateristics of control group as being sedentary since their total sitting time was higher than AS group.

Another significant results of our study was the association of one day physical activity behaviour and disease relatedvariables(disease activity, functionality). As the total score of BASDAl increased, total high physical activity time decreased paralel with previous studies. Arends et al. showed that 
high levels of physical activity were associated with low disease activity (23). Brophy et al. reported that people with moderate and low disease activity had a high level of physical activity (7). Fongen et al. compared the physical activity levels between healthy controls and AS patients (mean age 51.5 years with high disease activity and low disease activity), using the International Physical Activity Questionnaire-Long Form and found similar results (6).

In this study, we found that functionality was correlated with total moderate and high physical activity time in consistent with previous studies. O'Dwyer et al. found that there was a significant relationship between total physical activity score and BASFI scores (8). A high level of physical activity was found to be associated with good functionality by Brophy et al (7). In another study showed that, AS patients with reduced time spent in moderate and high physical activity levels had lower functionality (25).

The present study has some limitations that should be adresssed. Most importantly, the sample size was small and the medication was ongoing. Patients' physical activity could be assessed for another day, so that hartworne effect could be prevented. Included controls could have led to selection bias based on physical activity behaviours. Individuals who have different jobs characteristics should be included to avoid sedentary behaviours in control groups for the further studies investigating physical activity behaviour of AS patients.

\section{CONCLUSION}

In conclusion, regardless of disease activity and pain which are common symptoms in AS, kinesiophobia was present even the population was consisted of AS patients. One day physical activity monitoring in AS patients showed that they have tendency to spend time with sitting and lying, similar with healthy controls. They did not differ from controls by means of light and high level physical activity time. However, the moderate level physical activity time in AS group was considerably lower compared to healthy control group.

Kinesiophobia did not influence time spending physically active in AS patients. Further studies with larger sample size and longer time periods should be carried out to reveal physical activity behaviours and its related factors in AS patients who will survive with this disease for years.

\section{REFERENCES}

[1] Inanir A, Yigit S, Sariyildiz M, Sogut E. Outcomes of Turkish ankylosing spondylitis patients. Eur J Gen Med 2013;10(3): 145-149.

[2] Plasquı G, Bonen A, Geusens P. Physical activity and body composition in patients with ankylosing spondylitis. Arthritis Care Res 2012; 64(1):101-107.

[3] Caglar N, Burnaz Ö, Akın T, Ozgonenel L. Demographic and clinical properties and medical treatments of patients followed as ankylosing spondylitis. Istanbul Med J 2011;12(1):19-24.
[4] Akyol G, Ozalevli S, Ucan E. The relationship between pulmonary function and exercise capacity and quality of life in patients with ankylosing spondylitis. Tuberc Thorax 2013;61(3): 227-234.

[5] Kucharz E, Kotulska A, Kopec-Medrek M, Widuchouska M. Opinion of patients with ankylosing spondylitis on risk factors impairing their quality of life. Rheumatol Int 2013; 33: 28992901.

[6] Fongen C, Sveaas S, Dagfinrud H. Barriers and facilitators for being physically active in patients with ankylosing spondylitis: A cross-sectional comparative study. Musculoskeletal Care 2014;13:76-83.

[7] Brophy S, Cooksey R, Davies H, Dennis M, Zhou SM, Siebert $S$. The effect of physical activity and motivation on function in ankylosing spondylitis. A cohort study. Semin Arthritis and Rheum 2013;42(6): 619-626.

[8] O'Dwyer T, O'Shea F, Wilson F. Decreased physical activity and cardiorespiratory fitness in adults with ankylosing spondylitis: a cross-sectional controlled study. Rheumatol Int 2015;35:1863-1872.

[9] Kori SH MR, Todd D. Kinesophobia: a new view of chronic pain behaviour. Pain Management 1990;3:35-43.

[10] Oskay D, Tuna Z, Duzgun I, Elbasan B, Yavuz Y, Tufan A. Relationship between kinesiophobia and pain, quality of life, functional status, disease activity, mobility, and depression in patients with ankylosing spondylitis. Turk J Med Sci 2017;47:1340-1347.

[11] Nas K, Cevik R, Bozkurt M, Gur A, Sarac A. Relationship between clinical findings, quality of life and functional disability related to disease activity in patients with ankylosing spondylitis. Turk J Rheumatol 2011;26(1):29-37.

[12] Van der Weegen S, Essers H, Spreeuwenberg M, Verwey $\mathrm{R}$, Tange $\mathrm{H}$, de Witte L, Meijer K. Concurrent validity of the MOX activity monitor compared to the ActiGraph GT3X. Telemed J E Health. 2015;21(4):259-266.

[13] Yılmaz O, YakutY, Uygur F, Ulug N. Turkish version of the Tampa scale for kinesiophobia and its test-retest reliability. Physiother Rehabil 2011;22(1):44-49.

[14] Melzack R. The McGill Pain Questionnaire: major properties and scoring methods. Pain 1975;1(3):277-299.

[15] Yakut Y, Yakut E, Bayar K, Uygur F. Reliability and validity of the Turkish version short-form McGill pain questionnaire in patients with rheumatoid arthritis. Clin Rheumatol 2007;26(7):1083-1087.

[16] Akkoc Y, Karatepe AG, Akar S, Kirazli Y, Akkoc N. A Turkish version of the Bath Ankylosing Spondylitis Disease Activity Index: reliability and validity. Rheumatol Int 2005;25(4):280-284.

[17] Ozer HT, Sarpel T, Gulek B, Alparslan ZN, Erken E. The Turkishversion of the Bath Ankylosing Spondylitis Functional Index: reliability and validity. Clin Rheumatol. 2005;24(2):123128.

[18] Wouters E, van Leeuwen N, Bossema E, Kruize A, Bootsma H, Biljsma JW, Geenen R. Physical activity and physical activity cognitions are potential factors maintaining fatigue in patients with primary Sjögren's Syndrome. Ann Rheum Dis 2012; 71:668-673.

[19] Malfliet A, Oosterwijck J, Meeus M, Cagnie B, Danneals L, Dolphens M, Buyl R, Nijs J. Kinesiophobia and maladaptive coping strategies prevent in improvements in pain catastrophizing following pain neuroscience education in 
fibromyalgia/chronic fatigue syndrome:An explorative study. Physiother Theory Pract 2016; 33(8):653-660.

[20] Heuts P, Vlaeyen J, Roelofs J, de Bie R, Aretz K,van Weel C, van Schayck OC. Pain-related fear and daily functioning in patients with osteoarthritis. Pain 2004; 110: 228-235.

[21] Er G, Angın E. Determining the relationship of kinesiophobia with respiratory functions and functional capacity in ankylosing spondylitis. Medicine 2017;96: 29 e7486.

[22] Swinnen T, Vlaeyen J, Dankaerts W, Westhovens R, Vlam K. Activity limitations in patients with axial spondyloarthritis: A role for fear of movement and (re)injury beliefs. J Rheumatol 2018; 45(3): 357-366.

[23] Arend S, Hofman M, Kamsma Y,van der Veer E, Houtman PM, Kallenberg CG, Spoorenberg A, Brouwer E. Daily physical activity in ankylosing spondylitis validity and reliability of the IPAQ and SQUASH and the relation with clinical assessments. Arthritis Res Ther 2013;15(4):99-113.

[24] Felicio D, Pereira D, Queiroz B, da Silva JP, Leopoldino AA, Pereira LS. Kinesiophobia is not associated with disability in elderly women with acute low back pain: Back Complaints in the Elders (BACE) Brazil study results. The Spine Journal 2016;16:619-625.

[25] Van Genderen S, Boonen A, van der Heijde D, Heuft L, Luime J, Spoorenberg A, Arends S, Landewe R, Plasqui G. Accelerometer quantification of physical activity and activity patterns in patients withankylosing spondylitis and population controls. J Rheumatol: 2015;42; 2369-2375.

[26] Prkachin K, Schultz I, Hughes E. Pain behaviour and the development of pain-related disability: the importance of guarding. Clin J Pain 2007; 23:270-277.

[27] Swinnen TW, Scheers T, Lefevre J, Dankaerts W, Westovens R, de Vlam K.Physical activity assessment in patients with axial spondyloarthritis compared to healthy controls: A technologybased approach. PLoS ONE 2014;9(2):e85309.

[28] Vanhelst J, Milukoviç J, Bui-Xuan G, Dieu O, Blondeau T, Fardy P, Beghin L. Comparison of two ActiGraph accelerometer generations in the assessment of physical activity in free living conditions. BMC Res Notes 2012;5: 187-195.

How to cite this article: Aydin G, Demirbuken I, Kaya Mutlu E, Polat MG. Does Kinesiophobia Effect One Day Physical Activity Behaviour and Functionality of Young People with Ankylosing Spondylitis? Clin Exp Health Sci 2020;1:27-32. DOI:10.33808/clinexphealthsci.533527 